\title{
LUNA: Present status and future prospects
}

\author{
A. Caciolli (for the LUNA Collaboration)
}

University and INFN of Padua, Padua, Italy

\begin{abstract}
One of the main ingredients of nuclear astrophysics is the knowledge of the thermonuclear reactions responsible for powering the stellar engine and for the synthesis of the chemical elements. At astrophysical energies the cross section of nuclear processes is extremely reduced by the effect of the Coulomb barrier and often extrapolations are needed. The Laboratory for Underground Nuclear Astrophysics (LUNA) is placed under the Gran Sasso mountain. Thanks to the environmental background reduction provided by its position many reactions involved in hydrogen burning has been measured directly at astrophysical energies. Based on this progress, currently there are efforts in several countries to construct new underground accelerators. The exciting science that can be probed with these new facilities will be highlighted.
\end{abstract}

\section{Introduction}

Nuclear processes generate the energy that makes stars shine. Moreover they are responsible of the synthesis of the elements (and isotopes) in stars. As a matter of fact, hydrogen, helium and all isotopes until lithium and beryllium are synthesized during the Big Bang Nucleosynthesis (BBN). All other nuclei are produced during the different characteristic phases of the star evolution [1].

The understanding of these nuclear processes is the goal of nuclear astrophysics and, in particular, the knowledge of the nuclear cross-sections 
involved in that processes. At astrophysical energies the cross section is highly reduced by the effect of the Coulomb repulsion and the nuclear reactions can occur only via tunnel effect.

Due to these small cross section values, the rate of the reactions, characterized by a typical energy release of a few $\mathrm{MeV}$, is too low, down to a few events per year, in order to stand out from the laboratory background. In many cases it is not possible even to reach energy values close to the Gamow peak and extrapolations are needed, leading to substantial uncertainties. A way to handle that background problem is to go in an underground environment. As a matter of fact the natural shielding provided by an underground site will guarantee a reduction of the cosmic flux of orders of magnitude leading to the success of experimental nuclear physics. LUNA [2] is placed under the Gran Sasso National Laboratories of INFN. Two accelerators were used during years. First a $50 \mathrm{kV}$ accelerator (hereafter LUNA1) [3] and then a $400 \mathrm{kV}$ accelerator (hereafter LUNA2) [4].

Under the Gran Sasso Laboratory the muon flux is reduced by a factor $10^{6}$ and the neutron flux by a factor of $1000[5,6]$. Further background reduction in the region below $3 \mathrm{MeV}$ in the gamma spectrum can be achieved by implementing a shielding made by copper and lead [7]. A review of the results achieved by the LUNA collaboration will be presented in this paper combined with a discussion on the future projects for nuclear astrophysics in underground with a MV accelerator.

\section{Solar hydrogen burning}

Hydrogen burning in the Sun proceeds mainly by the proton-proton chain, with a $0.8 \%$ contribution from the carbon-nitrogen-oxygen cycle (CNO cycle) [8]. The basic processes are by now well understood, leading to the so-called standard solar model [9] that explains both helioseismological data and neutrino observations. The main uncertainty affecting this model, the solar neutrino puzzle, has been spectacularly solved by large neutrino detectors $[10-12$, e.g.] showing that the missing solar neutrinos have undergone flavour oscillation.

LUNA started its work studying the ${ }^{3} \mathrm{He}\left({ }^{3} \mathrm{He}, 2 \mathrm{p}\right){ }^{4} \mathrm{He}$ reaction since there was the discussion on a possible resonance at the Gamow peak energy [13, 14]. This study was done By using the LUNA1 $50 \mathrm{kV}$ accelerator this reaction was studied directly at the energy of the Gamow peak ruling out the resonance existence [15]. The ${ }^{2} \mathrm{H}(\mathrm{p}, \gamma)^{3} \mathrm{He}$ reaction, responsible for the production of ${ }^{3} \mathrm{He}$, was also studied at LUNA1 [16]. 
The neutrino fluxes emitted by the Sun are strictly correlated with the nuclear processes involved in the hydrogen burning. The LUNA2 program was focused on these processes achieving important results. The ${ }^{3} \mathrm{He}(\alpha, \gamma){ }^{7} \mathrm{Be}$ reaction was studied by using both the gamma prompt detection [17] and the activation techniques [18] finding a perfect agreement within the two methods. This result was important not only to reduce the systematics, but also to solve the discrepancy previously shown in experiments based on the two different techniques.

The CN neutrino fluxes are governed by the ${ }^{14} \mathrm{~N}(\mathrm{p}, \gamma){ }^{15} \mathrm{O}$ reaction. It is the bottleneck of the first $\mathrm{CNO}$ cycle and therefore the ${ }^{13} \mathrm{~N}$ and ${ }^{15} \mathrm{O}$ neutrinos are controlled by this reaction. LUNA deeply studied this reaction finding that the S-factor was a factor of two lower than what reported in the NACRE database [19]. This results are shown in [20] and reference therein.

A new precise knowledge of the ${ }^{14} \mathrm{~N}(\mathrm{p}, \gamma){ }^{15} \mathrm{O}$ cross section has been raised to solve the so called Solar Composition Problem [8]: the conflict between helioseismology and the new metal abundances (i.e. the amount of elements different from hydrogen and helium) that emerged from improved modelling of the photosphere [21]. As a matter of fact, the CNO neutrino flux is decreased by about $30 \%$ in going from the high to the low metallicity scenario. This way it will be possible to test whether the early Sun was chemically homogeneous [22], a key assumption of the standard Solar Model. In order to reduce the nuclear uncertainties in the solar model a new measurement was performed reaching the final value of $\mathrm{S}_{1,14}(0)=1.57 \pm 0.13 \mathrm{keV}$ barn $[23,24]$.

\subsection{Second, third CNO cycles, and the Mg-Al}

In recent years the LUNA collaboration focused its attention on several reactions involved in hydrogen burning Nova explosion. The first reaction studied in this program was the ${ }^{15} \mathrm{~N}(\mathrm{p}, \gamma){ }^{16} \mathrm{O}[25]$. This is the link from the first to the second $\mathrm{CNO}$ cycle and it was studied intensively at the LUNA accelerator. Two new experiments were performed by using ${ }^{15} \mathrm{~N}$ enriched solid targets ( [26] and reference therein). The LUNA measurements cover totally the Gamow peak for Nova explosion where the ${ }^{15} \mathrm{~N}(\mathrm{p}, \gamma){ }^{16} \mathrm{O}$ is important and the cross section was found to be lower than a factor of 2 with respect what reported in the NACRE database [19]. This leads to a reduction of ${ }^{16} \mathrm{O}$ produced by Novae of a $40 \%$ [26].

The ${ }^{17} \mathrm{O}(\mathrm{p}, \gamma){ }^{18} \mathrm{~F}$ reaction was investigated from 2011 to 2013 . In particular, the ratio between the reaction rates of ${ }^{17} \mathrm{O}(\mathrm{p}, \alpha){ }^{14} \mathrm{~N}(\mathrm{Q}=1.2 \mathrm{MeV})$ and ${ }^{17} \mathrm{O}(\mathrm{p}, \gamma){ }^{18} \mathrm{~F}(\mathrm{Q}=5.6 \mathrm{MeV})$ channels is one of the most important parameters for the galactic synthesis of ${ }^{17} \mathrm{O}$, the stellar production of radioactive 
${ }^{18} \mathrm{~F}$, and for predicted $\mathrm{O}$ isotopic ratios in presolar grains $[27,28]$.

Since the ${ }^{18} \mathrm{~F}$ is a radio-nuclide with a half life of $\approx 110 \mathrm{~min}$, the cross section has been derived both by detecting the prompt gamma rays and by counting the $511 \mathrm{keV} \gamma \mathrm{s}$ emitted by the ${ }^{18} \mathrm{~F}$ decay. The results are perfectly in agreement reducing considerably the systematic uncertainties [29]. The LUNA results affect not only the direct capture evaluation, but the $183.3 \mathrm{keV}$ resonance strength was also measured with a value of $\omega \gamma=(1.67 \pm 0.12) \mu \mathrm{eV}$. As a matter of fact, the LUNA measurements cover the whole Gamow peak referred to Nova scenarios reducing by a factor of 4 the uncertainty on this reaction in stellar models and in particular on the oxygen and fluorine isotopes produced in Nova explosions [29,30]. The very low uncertainty obtained in this experiment was possible thank to an intensive study of the target, realised and tested directly by the LUNA group with IBA and SIMS technique [31].

To measure also the ${ }^{17} \mathrm{O}(\mathrm{p}, \alpha){ }^{14} \mathrm{~N}$ reaction, a new chamber has been constructed which allows to place 8 silicons detectors in backward directions. The setup is described in [32] in details.

The study of the CNO cycles is the natural precursor for the hydrogen burning in $\mathrm{Ne}-\mathrm{Na}$ and $\mathrm{Mg}-\mathrm{Al}$ cycles. One of the reaction more interesting of the Ne-Na cycle is the ${ }^{22} \mathrm{Ne}(\mathrm{p}, \gamma)^{23} \mathrm{Na}$. This is one of the slowest reactions of the entire cycle and dominates the uncertainty for the production of many isotopes from neon to aluminum in different stellar scenarios. This is due to the presence of many resonances at stellar energies where only upper limits were given. With a setup characterized by two high purity germanium detectors fully shielded [33] this reaction has been studied and several resonance strengths measured reducing drastically the uncertainties on the reaction rate [34].

The problem of the ${ }^{26} \mathrm{Al}$ production is one of the most interesting cases [35]. LUNA measured precisely several resonances for ${ }^{24,25} \mathrm{Mg}(\mathrm{p}, \gamma)^{26,27} \mathrm{Al}$ in order to reduce the uncertainties on those reactions as requested in the astrophysical models $[36,37]$. The impact of the LUNA results is discussed in details in a recent work [38]. It is worth to mention that the ${ }^{26} \mathrm{Al}$ uncertainty in $\mathrm{C} / \mathrm{Ne}$ explosive burning is dominated by the ${ }^{25} \mathrm{Mg}(\alpha, \mathrm{n}){ }^{26} \mathrm{Al}$ reaction as discussed in [39] and reference therein.

\section{Big Bang nucleosynthesis}

The ${ }^{3} \mathrm{He}(\alpha, \gamma){ }^{7} \mathrm{Be}$ reaction has an important role in solving the problem of the Spite plateau [40]. LUNA measured this reaction in the Gamow 
peak for Big Bang Nucleosynthesis reducing the uncertainties to $3 \%$ overall. Another problem concerning lithium isotopes as been raised recently: the ${ }^{6} \mathrm{Li}$ has been measured to be 3 order of magnitude higher than what expected in BBN $[41,42]$. For ${ }^{6} \mathrm{Li}$ production in the Big Bang, the main nuclear physics unknown is the ${ }^{2} \mathrm{H}(\alpha, \gamma){ }^{6} \mathrm{Li}$ reaction rate. The setup used to study this reaction is already described in details [43]. A long and detailed study of this background was required in order to perform the analysis of the data $[43,44]$. This way a possible nuclear solution of the ${ }^{6} \mathrm{Li}$ problem has been ruled out by LUNA.

\section{Science case for a future higher-energy accelerator underground}

Recent advances in astronomy and astrophysics require nuclear data at energies that are higher than the high-energy limit of LUNA2.

Most notably, the ${ }^{12} \mathrm{C}(\alpha, \gamma){ }^{16} \mathrm{O}$ reaction still eludes experimental and theoretical efforts to pin down its precise rate. This reaction, together with the triple- $\alpha$ reaction, determines the ratio of carbon to oxygen at the end of helium burning, a value that has wide-ranging impacts on the nucleosynthesis of heavier elements.

Whereas a direct ${ }^{12} \mathrm{C}(\alpha, \gamma){ }^{16} \mathrm{O}$ study at the relevant energy of $300 \mathrm{keV}$ is impossible due to the forbiddingly-low absolute yield, a study in a lowbackground environment such as LUNA at higher energies can help improve necessary extrapolations by providing constraints at energies where there are currently no data. The study of this reaction is based on a precise knowledge of the targets, since the background induced by the parasitic $(\alpha, \gamma)$ reaction on ${ }^{13} \mathrm{C}$ can overwhelm the signal of the ${ }^{12} \mathrm{C}(\alpha, \gamma){ }^{16} \mathrm{O}$ reaction if the isotopic ratio ${ }^{12} \mathrm{C} /{ }^{13} \mathrm{C}$ is less than $10^{5}$ (at least three order of magnitude higher than in natural carbon). The LUNA collaboration has started a deep investigation on ${ }^{12} \mathrm{C}$ enriched targets and their stability, by performing analysis tests on different backings and cleaning techniques and to understand the behaviour of the produced targets against irradiated charge. Those tests are performed at the Laboratori Nazionali di Legnaro and they will continue also in 2015 in order to have a complete understanding of the targets and in order to keep better under control the production techniques used in their creation. This work is essential for the success of the ${ }^{12} \mathrm{C}(\alpha, \gamma){ }^{16} \mathrm{O}$ cross section measurements.

Another important open issue of nuclear astrophysics is the neutron source reactions. In particular the ${ }^{13} \mathrm{C}(\alpha, \mathrm{n}){ }^{16} \mathrm{O}$ and ${ }^{22} \mathrm{Ne}(\alpha, \mathrm{n})^{25} \mathrm{Mg}$ reac- 
tions. They are responsible for the production on neutrons involved in the slow neutron capture process, called the astrophysical s-process. Whereas those reactions are the subject of intensive experimental study, so far the reactions actually producing the neutrons have not yet been measured in the relevant energy range since they should be addressed by an underground accelerator.

A third topic is to complement some of the proton- and $\alpha$-capture reactions studied at the LUNA2 accelerator at higher energy. Such a continuation is particularly important for the Big Bang reactions ${ }^{3} \mathrm{He}(\alpha, \gamma){ }^{7} \mathrm{Be}$ and ${ }^{2} \mathrm{H}(\alpha, \gamma)^{6} \mathrm{Li}$, where the present LUNA2 $400 \mathrm{kV}$ machine can only cover the lower part of the relevant energy region.

\section{Future underground accelerator facilities}

Based on the successes of the LUNA collaboration, around the world several efforts are underway to install high-current, stable-beam accelerators in underground sites. LUNA-MV project was started in order to install a $3 \mathrm{MV}$ machine in the underground laboratories of Gran Sasso. The new accelerator has already been financed by the Italian government and it should be installed in the next years at the Gran Sasso Laboratory. The synergy between the existing LUNA2 and the new LUNA-MV accelerator will allow to perform reaction studies in a wide range of energies with complete understanding of the setups involved. The planned DIANA facility at the Deep Underground Science Laboratory DUSEL in the Unites States also includes a megavolt and a lower-energy machine. Another project is under discussion at the Canfranc underground laboratory in the Pyrenees, Spain. As part of a staged approach, even an accelerator laboratory in a shallow-underground facility such as Felsenkeller (Dresden, Germany) is under consideration.

At present, the existing $400 \mathrm{kV}$ LUNA2 machine continues its scientific program outlined here. The next few years will show where this highly successful approach will eventually be complemented by one or more higherenergy accelerators underground. The technique is sufficiently mature to address not only the data needs of the astrophysics community, but it has the potential to benefit also the astroparticle and other communities.

\section{Acknowledgments}

The work at LUNA is carried out in the framework of the LUNA collaboration; see e.g. Ref. [43] for an author list. Financial support by OTKA 
K101328, NN83261, DFG (BE 4100/2-1), and NAVI is also gratefully acknowledged.

\section{References}

[1] C. Iliadis, Nuclear Physics of Stars, Wiley-VCH, 2007.

[2] C. Broggini, D. Bemmerer, A. Guglielmetti, and R. Menegazzo, Annu. Rev. Nucl. Part. Sci. 60, 53 (2010).

[3] U. Greife et al., Nucl.Instr.Meth. A 350 (1994) 327.

[4] A. Formicola et al., Nucl.Instr.Meth. A 507 (2003) 609.

[5] D. Bemmerer, et al., Eur. Phys. J. A 24, 313-319 (2005).

[6] T. Szücs, et al., Eur. Phys. J. A 44, 513 (2010).

[7] A. Caciolli, et al., Eur. Phys. J. A 39, 179-186 (2009).

[8] A. M. Serenelli, S. Basu, J. W. Ferguson, and M. Asplund, Astrophys. J. Lett. 705, L123-L127 (2009), 0909.2668.

[9] J. N. Bahcall, and M. H. Pinsonneault, Phys. Rev. Lett. 92, 121301 (2004).

[10] J. Hosaka, et al., Phys. Rev. D 73, 112001-+ (2006).

[11] I. Ahmad, J. P. Greene, E. F. Moore, S. Ghelberg, A. Ofan, M. Paul, and W. Kutschera, Phys. Rev. C 74, 065803-+ (2006).

[12] C. Arpesella, et al., Phys. Rev. Lett. 101 (2008).

[13] W.A. Fowler, Nature 238 (1972) 24

[14] V.N. Fetysov and Y.S. Kopysov, Phys. Lett. B 40 (1972) 602

[15] R. Bonetti, et al., Phys. Rev. Lett. 82, 5205-5208 (1999).

[16] C. Casella, et al., Nucl. Phys. A 706, 203-216 (2002).

[17] D. Bemmerer, et al., Phys. Rev. Lett. 97, 122502 (2006).

[18] F. Confortola, et al., Phys. Rev. C 75, 065803-+ (2007).

[19] C. Angulo et al. (NACRE Coll.), Nucl. Phys. A 656 (1999) 3. 
[20] D. Bemmerer, et al., Nucl. Phys. A 779, 297-317 (2006).

[21] C. Pena-Garay and A.M. Serenelli, arXiv: 0811.2424 (2008.)

[22] W.C. Haxton and A.M. Serenelli, arXiv:0902.0036 (2009).

[23] M. Marta, et al., Phys. Rev. C 78, 022802(R) (2008).

[24] M. Marta, et al., Phys. Rev. C 83, 045804 (2011).

[25] D. Bemmerer, et al., J. Phys. G 36, 045202 (2009).

[26] A. Caciolli, et al., A\&SA 533, A66 (2011).

[27] C. Fox et al., Phys. Rev. C 71 (2005) 055801.

[28] J.R. Newton et al., Phys. Rev. C 81 (2010) 045801.

[29] D. A. Scott, et al., Phys. Rev. Lett. 109, 208001 (2011).

[30] A. Di Leva, et al., Phys. Rev. C 89, 015803 (2014).

[31] A. Caciolli, et al., Eur. Phys. J. A 48, 144 (2012).

[32] C. G. Bruno, et al., Eur. Phys. J. A 51, 94 (2015).

[33] F. Cavanna, et al., Eur. Phys. J. A 50179 (2014).

[34] F. Cavanna, et al., submitted to PRL.

[35] C. Iliadis, et al., Astrophys. J. Supp. Series 193, 16 (2011).

[36] B. Limata et al., Phys. Rev. C 82 (2010) 015801

[37] F. Strieder et al., Phys. Lett. B 707, 60 (2012).

[38] O. Straniero et al., Astrophys. J. 763, 100 (2012).

[39] A. Caciolli, et al. Eur. Phys. J. A 50, 147 (2014).

[40] M. Spite, and F. Spite, Nature 297, 483-485 (1982).

[41] V. Smith, D. Lambert, and P. Nissen, Astrophys. J. 408, 262 (1993).

[42] M. Asplund, et al., Astrophys. J. 644, 229-259 (2006).

[43] M. Anders et al., Eur. Phys. J. A 49, 28 (2013).

[44] M. Anders et al., Phys. Rev. Lett. 113, 042501 (2014). 